Advances in Gene Technology: The Genome and Beyond -

Structural Biology for Medicine (Proceedings of the 2002 Miami

Nature Biotechnology Winter Symposium)

TheScientificWorld 2002, 2(S2), 55-56

ISSN 1532-2246; DOI 10.1100/tsw.2002.28

\title{
BIOCHEMICAL AND STRUCTURAL ANALYSIS OF THE ROLE OF THE WLB GENE LOCUS IN BORDETELLA PERTUSSIS LIPOPOLYSACCHARIDE BIOSYNTHESIS
}

\author{
$\underline{\text { Velupillai S. Kannathasan }}^{\mathrm{a}}$, Chang J. Dong ${ }^{\mathrm{b}}$, Corin Wing ${ }^{\mathrm{a}}$, Andrew Preston ${ }^{\mathrm{c}}$, Duncan J. Maskell ${ }^{\mathrm{c}}$, \\ James H. Naismith ${ }^{\mathrm{b}}$, and Robert A. Field ${ }^{\mathrm{a}, *}$ \\ ${ }^{a}$ Centre for Carbohydrate Chemistry, University of East Anglia, Norwich, NR4 7TJ, U.K.; ${ }^{b}$ Centre for \\ Biomolecular Sciences, University of St Andrews, KY16 9ST, U.K.; 'School of Veterinary Medicine, \\ University of Cambridge, Cambridge, CB3 OES, U.K. \\ *r.a.field@uea.ac.uk
}

INTRODUCTION. Bordetella pertussis is the causative agent of whooping cough in children and chronic cough in adults. Bordetella lipopolysaccharide (LPS) has been implicated in the infection process and has been shown to be highly immunogenic, and acts as an immunological adjuvant which displays the properties of an endotoxin. Tentative gene function assignments have been made for the B. pertussis LPS band A trisaccharide biosynthetic locus ( $w l b$ ), albeit based on rather weak sequence similarities[1,2]. The $B$. pertussis $w l b D$ gene product is a putative uridine-5diphosphate $\mathrm{N}$-acetylglucosamine 2'-epimerase based on its homology to E. coli rffE (32\% identical), an established UDP-GlcNAc 2'-epimerase that is involved in Enterobacterial Common Antigen (ECA) formation. The key active site residues in rffE are present in the $w l b D$ sequence.

RESULTS AND DISCUSSION. The wlbD gene from B. pertussis has been cloned and overexpressed in E. coli and the resulting protein has been purified to homogeneity. All the activity assays failed to show the epimerase activity in vitro and wlbD failed to complement the E. coli $r f f E$ mutant. This suggests that assignment of $w l b D$ is almost certainly wrong, which prompts reexamination of the entire biosynthetic pathway (see Fig.1).The most likely substrate for $w l b D$ may be UDP-Glc-2,3-diNAc. The 3-D structure of wlbD may throw light on its function, crystals of the mutant Gln339Arg wlbD enzyme have been obtained in the presence and absence of UDPGlcNAc and $1.6 \AA$ (in Grenoble, France) and $2.3 \AA$ (in house) data sets were collected, respectively.[4] In order to solve the structure a Seleno-methionine variant protein was prepared and MAD data were collected since molecular replacement using rffE as model failed. The crystal structure exists as a dimer and each monomer has two domains (see Fig. 2). The wlbD structure shares marked topological similarity to the rffE structure and other glycosyltransferases (DALI analysis). Further structural analysis is going on. 

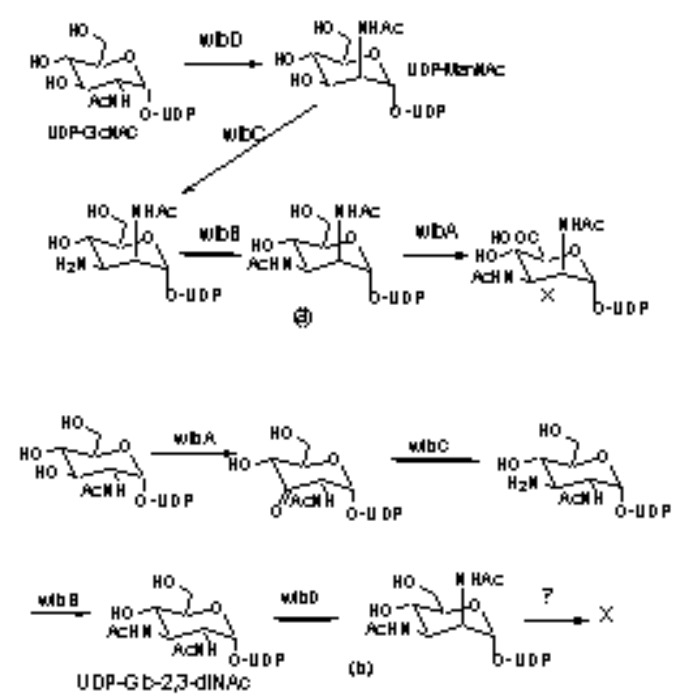

FIGURE 1. (a) Original proposed pathway; (b) revised pathway.

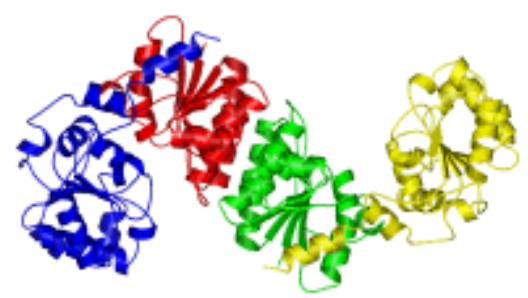

FIGURE 2. Crystal structure of wlbD.

ACKNOWLEDGEMENT. This work was supported by the UK Biotechnology and Biological Sciences Research Council.

\section{REFERENCES}

1. Allen, A.G. and Maskell, D.J. (1996) Mol. Microbiol. 19, 37-52.

2. Allen, A.G., Thomas, R.M., Cadisch, J.T., and Maskell, D.J. (1998) Mol. Microbiol. 29, 27-38.

3. Campbell, R.E., Mosimann, S.C., Tanner, M.E., and Strynadka, N.C.J. (2000) Biochemistry 49, 14993-15001.

4. Kannathasan, V.S., Staines, A.G., Dong, C.J., Preston, A.G., Maskell, D.J., Naismith, J.H., and Field, R.A. (2001) Acta Crystallogr. D57, 1310-1312. 